Bundesgesundheitsbl $2011 \cdot 54: 789-790$

DOI 10.1007/s00103-011-1311-4

Online publiziert: 28. Juni 2011

(c) Springer-Verlag 2011

\author{
R. Seitz ${ }^{1}$ C.K. Schneider ${ }^{1} \cdot$ H. Hengel ${ }^{2}$ \\ ${ }^{1}$ Paul-Ehrlich-Institut, Langen \\ ${ }^{2}$ Universität Düsseldorf, Düsseldorf
}

\title{
Arzneimittel für neuartige Therapien
}

\author{
Wissenschaftliche, medizinische, \\ ökonomische und ethische Aspekte
}
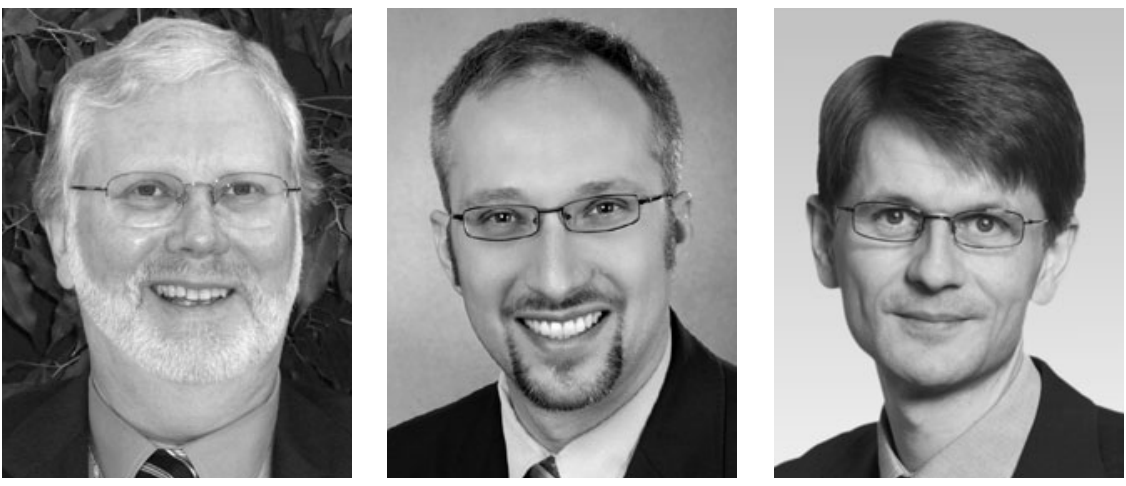

und anderer europäischer Gesetze konnte die bisherige Praxis in Mitgliedstaaten mit fehlender oder wenig eingreifender Regulierung von zellbasierten innovativen Therapien nicht weitergeführt werden. De facto kam es zu einer eingreifenden Intensivierung der behördlichen Kontrolle durch die neuen verbindlichen Rahmenbedingungen, die unter anderem eine zentrale europäische Zulassung von ATMP vorsehen.

In dem vorliegenden zweiten Themenheft zu ATMP soll beleuchtet werden, wie sich das Feld unter diesen gesetzlichen und behördlichen Rahmenbedingungen weiterentwickelt hat. Im Fokus sollen dabei aktuelle wissenschaftliche Erkenntnisse und deren Umsetzung in die medizinische Praxis stehen. Allerdings kann sich auch dieses wissenschaftlich spannende Feld nicht losgelöst von ökonomischen Gegebenheiten entwickeln. Zudem sind auch gerade im Zusammenhang mit den ATMP, an die große Hoffnungen, aber auch Bedenken geknüpft werden, ethische Fragen zu erörtern.

Im ersten Beitrag beschreiben H. Bönig, M. Heiden, J. Schüttrumpf, M.M. Mül- ler und E. Seifried das Potenzial der schon lange für die Regeneration der Knochenmarksfunktion nach Hochdosistherapie von Malignomen genutzten hämatopoetischen (blutbildenden) Stammzellen als Vorläuferzellen verschiedener Differenzierungsgrade können durch Punktion des Knochenmarkes, nach Mobilisierung durch Wachstumsfaktoren mittels Apherese aus dem peripheren Blut sowie aus dem Nabelschnurblut gewonnen werden. Die Spannweite der Entwicklungen reicht von nicht substanziell bearbeiteten Präparationen für neue Indikationen in der Geweberegeneration bis hin zu aufwendig hergestellten Zellund Gentherapeutika. Über die Klinik und Diagnostik von Gelenkknorpelschäden, die praktisch keine Selbstheilungstendenz zeigen, und die Möglichkeiten ihrer Behandlung mithilfe autologer Vorläuferzellen im Vergleich zu anderen Therapieoptionen berichten $M$. Steinwachs, Ph. Niemeyer und B. Waibl.

Auf das vorherige Themenheft aufbauend, stellen C. Walter, B. Rohde, D.C. WiAusgangsmaterial für ATMP. Stamm- und wirklicht werden. Aufgrund der sogenannten ATMP-Verordnung (EG) 1394/2007 
cke, C. Pohler, A. Lührmann und H. von der Leyen dar, wie unter den komplexen regulatorischen Rahmenbedingungen die heterogenen und oft sehr anspruchsvollen Ansätze zu neuen Therapien entwickelt werden können. Hierzu sollte der Geburt und Reifung der Ideen im Labor ein gut durchdachtes präklinisches und klinisches Entwicklungsprogramm folgen. Da hier häufig aus der akademischen Forschung heraus gegründete kleine Unternehmen mit wenig regulatorischer Erfahrung agieren, empfehlen die Autoren, frühzeitig die Beratung durch die zuständigen Behörden zu suchen. In Deutschland ist das Paul-Ehrlich-Institut (PEI) für die ATMP zuständig, zum Beispiel für die Genehmigung klinischer Studien. Die Zulassung und wichtige Verfahren im Vorfeld liegen bei der European Medicines Agency (EMA) in London, wobei auch hier die Behörden der Mitgliedstaaten wie das PEI eine wichtige Rolle spielen. Eine der ersten Fragen wird häufig sein, ob das beabsichtigte therapeutische Produkt überhaupt regulatorisch als ATMP einzustufen ist. Um in dieser Frage frühzeitig Rechtssicherheit zu schaffen, wurde bei der EMA in London ein kostenfreies Verfahren etabliert. Die Klassifizierung durch das Committee for Advanced Therapies (CAT), die innerhalb von 60 Tagen erfolgen soll, kann in jeder Phase der Entwicklung eines ATMP beantragt werden. C. Voltz-Girolt, P. Celis, M. Boucaumont, L. D'Apote, M.-H. Pinheiro und M. Papaluca-Amati stellen die bisherigen Erfahrungen und die positiven Auswirkungen der Klassifizierung für Antragsteller und Behörden vor. Dabei wird häufig der erste Kontakt zur EMA aufgebaut, der weitere Schritte erleichtert, wie zum Beispiel einen Antrag auf Ausweisung als Orphan Medicinal Product (Arzneimittel für seltene Leiden), womit einige Anreize verbunden sind. Eine weitere, gerade für relativ junge, kleine und mittelständische Unternehmen potenziell interessante Option ist die Zertifizierung neuartiger Therapien durch die EMA. Wie A. Berger, S. Schüle und E. Flory darlegen, stellt diese eine Art Gütesiegel für die Produktentwicklung dar, das die Attraktivität für potenzielle Investoren und Lizenznehmer steigern kann.

Auch wenn verschiedene Mechanismen der regulatorischen Beratung und Unterstützung vorgesehen sind, bleibt die Ent- wicklung von ATMP auf dem Weg zur Anwendung am Patienten eine große Herausforderung. M. Reiss, I.C. Büttel und C.K. Schneider berichten über Erfahrungen aus dem CAT und stellen potenzielle Fallstricke auf dem Weg vom Konzept zur klinischen Anwendung neuartiger Therapien dar. Ž. Buljovčić schildert in ihrem kritischen Beitrag den langen Weg zur zentralen Zulassung aus der Sicht kleiner Biotechnologieunternehmen. Für solche Firmen sind der Umfang der Anforderungen und der Zeitrahmen zu deren Erfüllung vielfach kritisch. In diesem Zusammenhang diskutiert Frau Buljovčić den $\$ 4 b$ des Arzneimittelgesetzes, der für ATMP eingeführt wurde, ,die noch nicht in ausreichender Anzahl hergestellt worden sind, sodass die notwendigen Erkenntnisse für ihre umfassende Beurteilung noch nicht vorliegen" - hier sieht das Arzneimittelgesetz unter bestimmten Voraussetzungen eine Genehmigung durch das PEI vor.

Wie generell bei der Entwicklung neuer Arzneimittel ist es auch bei den ATMP das Ziel, die Behandlung von Patienten zu verbessern, insbesondere bei prognostisch bisher ungünstigen Erkrankungen. Es ist daher folgerichtig, wenn sich Patientenvertreter bei der Entwicklung von ATMP einbringen, wie von F. Bignami, A.J. Kent, M. Lipucci di Paola und N. Meade beschrieben. So wurde bei der EMA mit der Patients and Consumers Working Party (PCWP) ein institutionalisiertes Forum für den Dialog zwischen Behörden und Patientenorganisationen geschaffen. M. Thanner und E. Nagel geben eine umfassende medizinethische Bewertung der ATMP und eine Übersicht der Problemfelder und Lösungsansätze. Hier wird angesichts der gerade bei den ATMP teils immensen Erwartungen und des auch innerhalb Europas inhomogenen gesellschaftlichen Hintergrundes dem ethischen Diskurs eine wichtige Rolle zukommen.

Am Ende des langen und schwierigen Weges besteht das legitime Interesse, das entwickelte ATMP vermarkten zu können. A. P. F. Ehlers und A. Wenke diskutieren die Erstattungsfähigkeit neuartiger Arzneimitteltherapien im deutschen Gesundheitssystem. Das Spektrum wird abgerundet durch den Beitrag von B. Ziegele, L. Dahl und A.T. Müller, die das Innovationsbüro am Paul-Ehrlich-Institut als ein Ange- bot zur regulatorischen Begleitung der Entwicklung neuartiger Therapien vorstellen.

Die in diesem Themenheft vorgelegte Standortbestimmung zeigt, dass sich das Feld der ATMP nach wie vor in einem $d y$ namischen Entwicklungsprozess befindet. Es entstehen weiterhin wissenschaftlich anspruchsvolle Konzepte, die durch hohe Standards hinsichtlich Qualität und Konsistenz der Herstellung und durchdachte und kompetent umgesetzte präklinische und klinische Entwicklungsprogramme bis zur Anwendung in der medizinischen Praxis geführt werden müssen. $\mathrm{Zu}$ dem müssen ATMP, um erfolgreich zu sein, auch ethischen Erwägungen genügen und die Hürden zur Erstattungsfähigkeit überwinden. Die durch die Europäische Gesetzgebung vereinheitlichten hohen regulatorischen Anforderungen werden gerade von relativ jungen, kleinen und mittelständischen Unternehmen als eine sehr große Belastung gesehen. Daher sollte die Zukunft des einerseits faszinierenden und zukunftsträchtigen, andererseits komplexen und herausfordernden Themas ATMP nicht unkritisch, aber doch mit einigem Optimismus gesehen werden.

Ihre

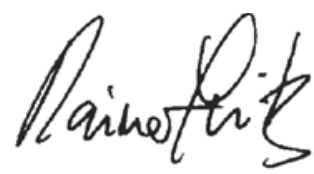

Rainer Seitz

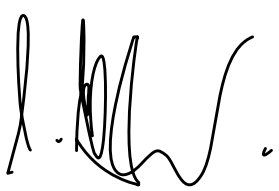

Christian Schneider

\section{Hatmur fenen}

Hartmut Hengel

\section{Korrespondenzadresse \\ Prof. Dr. R. Seitz}

Paul-Ehrlich-Institut

Paul-Ehrlich-Str. 51-59, 63225 Langen

seira@pei.de 